\title{
Heartbeat: Imaging complex vascular anatomy in congenital heart disease
}

Pulmonary hypertension in children negatively impacts clinical outcome and is not always responsive to medical therapy. An entire supplement to Heart ${ }^{1-3}$ ) provides several articles with detailed reviews and consensus statements for management of pediatric pulmonary hypertension. A novel approach to management of pediatric patients with refractory pulmonary hypertension is creation of a shunt from the descending aorta to the left pulmonary artery to unload the suprasystemic pulmonary vascular circuit by shunting into the systemic circulation, often called a "reverse Potts shunt" because the flow direction is the opposite of the shunt created to increase pulmonary blood flow in other types of congenital heart disease. However, creation of this shunt by a transcatheter approach requires advanced cardiac imaging to ensure the two vessels have enough contact area for a successful procedure (figure 1). The imaging approach for this procedure was evaluated in detail in a series of patients by Sizarov and colleagues (see page 1735).

Schranz (see page 1699) comments that this new approach is promising but "Still, there is an intense 'learning curve' before we reach the point where the safety and efficacy of a transcatheter Potts shunt creation will overcome the high risk of the surgical approach. However, I am fully convinced that the transcatheter approach creating a right-left shunting Potts communication will further augment the therapeutic options for children and young adults with suprasystemic PAH. Highly sophisticated detailed haemodynamic and 'pre'-procedural imaging data are the prerequisite for success."

In a state-of-the-art review article (see page 1703) Khairy summarises the evidence and challenges in management of ventricular arrhythmias and sudden cardiac death in adult congenital heart disease (ACHD) patients. Although we know that ACHD patients have an increased risk of sudden death, our evidence base for informing decisions about primary prevention with an implantable

Correspondence to Professor Catherine M Otto, Division of Cardiology, University of Washington, Seattle,WA 98195, USA; cmotto@u.washington.edu cardiac defibrillator (ICD) is weak. Some data exists for tetralogy of Fallot patients ${ }^{4}$ but indications for other types of complex ACHD are based largely on expert opinion alone. Furthermore, ICD placement can be challenging due to complex anatomy with surgical conduits and baffles, obstructed venous access, intracardiac shunts, and systemic right ventricles or univentricular hearts, often necessitating custom solutions in each patient. On the other hand, advanced imaging and electrophysiological mapping (figure 2) have greatly improved our ability to identify and treat the arrhythmia mechanism in ACHD patients.

In an editorial commentary, Schmidt and Bhatt (see page 1701) argue that the standard research model of randomized controlled clinical trials is difficult to apply in ACHD because of the diverse diagnoses, variable surgical procedures, and relatively small patient numbers. They suggest alternate approaches that utilize direct patient and electronic medical record data. "PCORNet is an example of infrastructure that does just that; it is a research network organised by the Patient-Centered Outcomes Research Institute as an innovative partnership between healthcare system-based networks (termed clinical data research networks, containing institutional electronic medical records) and patient-centred networks (termed patient-powered research networks, such as advocacy groups, with data shared voluntarily by patients), brought together by a coordinating centre." Further, they argue that "we should encourage patients to be more involved in trial design and recruitment through organisations such as the Adult Congenital Heart Association and advocate with them
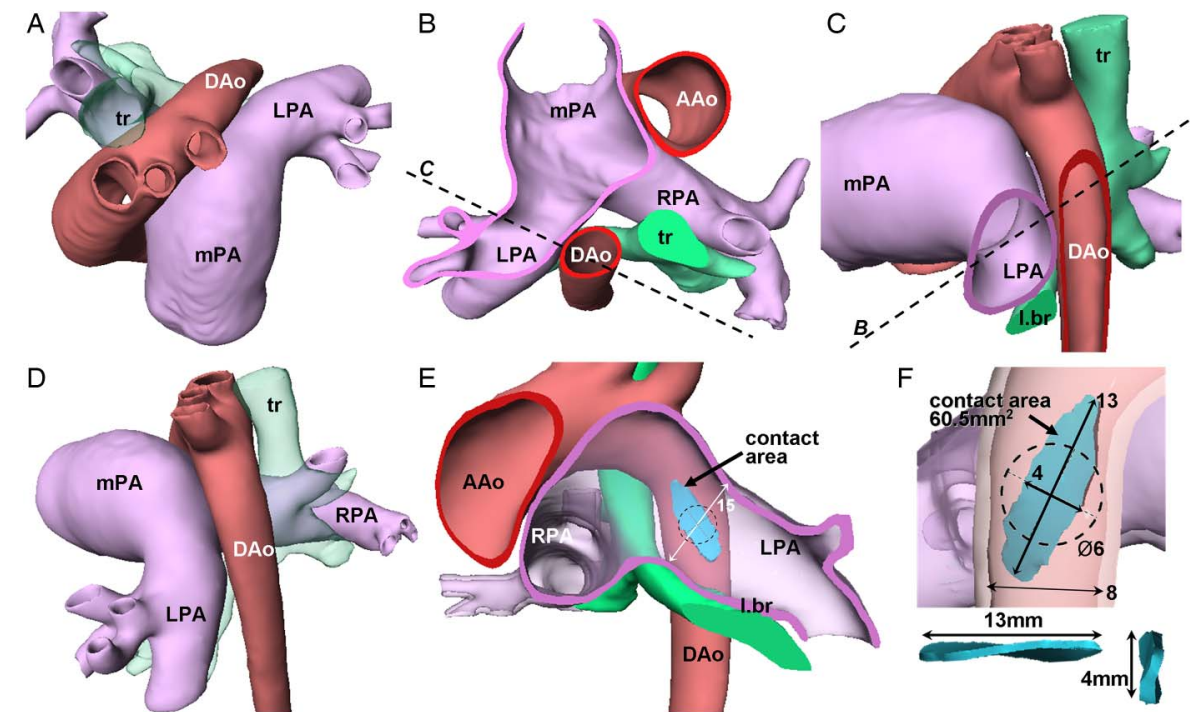

Figure 1 Spatial relationship of the vascular walls in a representative patient with severe suprasystemic pulmonary arterial hypertension (PAH). Aorta is colour-coded as red, pulmonary artery as purple and the trachea-bronchi as light-green. $(A)$ is a cranial view and (D) is the left lateral view of the whole three-dimensional (3D) model. (B) is the craniodorsal view of the transverse cut and $(C)$ is left lateral view of the oblique sagittal cut through the left pulmonary artery (LPA) and descending aorta (DAo). Such arrangement with the very short distance between two vessels was the most prevalent among the population of this study. ( $E$ and $F$ ) are oblique sagittal cuts through the LPA and DAo, respectively, and show the tightest contact area (colour-coded as light-blue) with the dimensions as indicated (in $\mathrm{mm}$ ). Note the elliptic shape of the tightest contact area with the longest diameter stretched along the DAo length and the LPA width. The dashed circle represents the cross-section of the presumably suitable stent with diameter of $6 \mathrm{~mm}$ being superimposed upon the tightest contact area. Note the differences in the playing space to get the catheter tip at the centre of the tightest contact area in horizontal and vertical planes. 


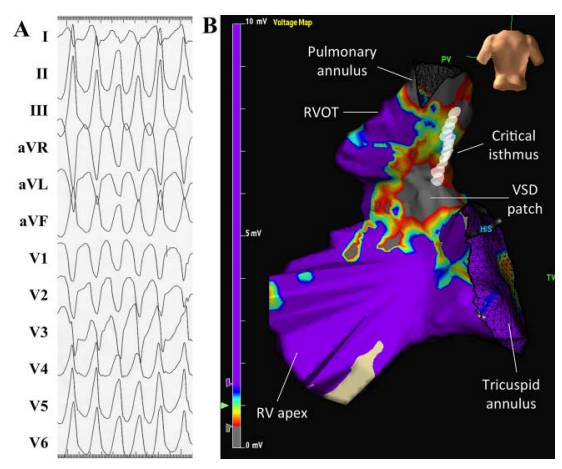

Figure 2 Ventricular tachycardia in tetralogy of Fallot. Shown in (A) are surface electrocardiographic recordings during ventricular tachycardia in a patient with tetralogy of Fallot. The tachycardia had a left bundle branch block morphology and inferior QRS axis (ie, positive in leads II, III and aVF).

(B) Shows a posterior view of a voltage map of the right ventricle (RV) using a

three-dimensional electroanatomic mapping system. Normal voltages are coloured purple and dense scar grey. The site of the ventricular septal defect (VSD) patch is indicated. The ventricular tachycardia was dependent on a critical corridor of tissue in the RV outflow tract (RVOT), which was transected by radiofrequency catheter ablation between the VSD patch and pulmonary annulus. Ablation lesion sites are indicated by white circles.

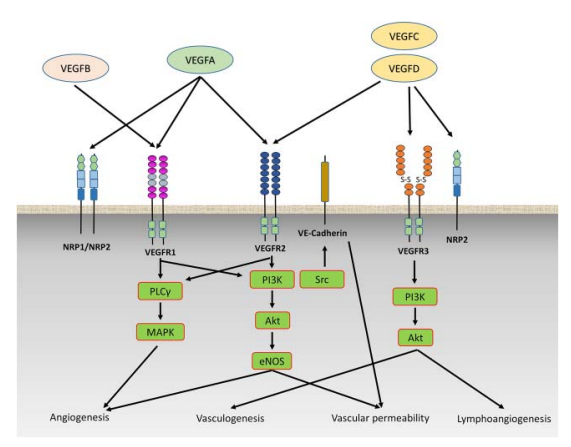

Figure 3 Cartoon illustrating the VEGF isoforms and their receptors. Recapitulative scheme of different VEGF isoforms and their receptors. Akt, Protein Kinase B; eNOS, endothelial nitric-oxide synthase; MAPK, mitogenactivated protein kinases; NRP, Neuropilin; PI3K, phosphatidylinositol-4,5-bisphosphate 3-kinase; PLC $\gamma$, phospholipase C gamma; VEGF, vascular endothelial growth factor; VEGFR, VEGF receptor; Src, Proto-oncogene tyrosine-protein kinase Src.

that wearable medical technology, such as cardiac rhythm monitors, should be able to seamlessly upload the data into both

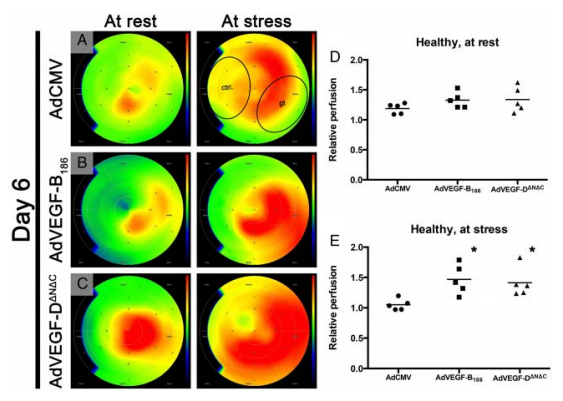

Figure 4 AdVEGF-B ${ }_{186}$ and AdVEGF-D $D^{\triangle N \Delta C}$ increase perfusion in healthy pig myocardium. Gene transfers were made to the posterolateral wall of the left ventricle, which corresponds to the lower right sector of the perfusion pictures, indicated by ellipse labelled gt. Colour scale is absolute; darkest blue is $0 \mathrm{~mL} / \mathrm{min} / \mathrm{g}$, green is $1,5 \mathrm{~mL} / \mathrm{min} / \mathrm{g}$ and deepest red is $3.0 \mathrm{~mL} / \mathrm{min} / \mathrm{g}$ or over. (A) Perfusion maximum is not localised in the gene therapy area in the control group indicating no effect of the control treatment. ( $B$ and $C$ ) The peak perfusion of both AdVEGF-B ${ }_{186}$ and AdVEGF- $D^{\triangle N \Delta C}$ is localised in the gene transfer area and the changes are even more pronounced at stress. ( $D$ and $E$ ) Relative perfusion was at rest $12 \%$ and at stress $40 \%$ higher for AdVEGF-B ${ }_{186}$ and at rest $13 \%$ and at stress $34 \%$ higher for AdVEGF-D ${ }^{\Delta N \Delta C}$ than for AdCMV. AdCMV, Ad-cytomegalovirus; ctrl, control anteroseptal area; gt, posterolateral gene transfer area.

their institutions' electronic medical records and registries.”

An experimental study in this issue addresses angiogenic gene therapy using new members of the vascular endothelial growth factor (VEGF) family (see page 1716) (figure 3). In this model, 'both AdVEGF-B $_{186}$ and AdVEGF-D ${ }^{\Delta \mathrm{N} \Delta \mathrm{C}}$ gene transfers induced efficient angiogenesis in the myocardium resulting in an increased myocardial perfusion measured by PET. Importantly, local perfusion increase did not induce any coronary steal effect." (figure 4). The authors conclude that "both treatments seem suitable new candidates for the induction of therapeutic angiogenesis for the treatment of refractory angina."

Mangialardi and Madeddu (see page 1697) suggest that additional studies are needed before these findings can be translated into a clinical trial, specifically the use of a model with normal coronary arteries, rather than atherosclerotic coronary disease, and the short follow-up period making it uncertain whether these effects will be maintained long-term. "Nevertheless, this report opens promising opportunities that may overcome the limits of traditional angiogenesis gene therapy and have far-reaching and critical impact on regenerative medicine for the treatment of ischaemic diseases."

The Education in Heart article in this issue (see page 1763) provides all the information needed to set up a stress echocardiography service. Excellent tables summarize the requirements for a stress echo service, including space, equipment and clinical staff, as well as indications for the procedure, types of stress testing and contraindications to the procedure. This practical article will be of great value to centers who already have or are considering a stress echocardiography service.

The Image Challenge in this issue (see page 1762) shows multimodality imaging demonstrating an unusual finding in a 57-year old woman with new onset hypertension and a diastolic murmur. Looking at these images, making the diagnosis, and reading the explanation of the findings will enhance your clinical expertise.

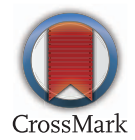

To cite Otto CM. Heart 2016;102:1695-1696.

Heart 2016;102:1695-1696.

doi:10.1136/heartjnl-2016-310600

\section{REFERENCES}

1 Hansmann G, Apitz C. Treatment of children with pulmonary hypertension. Expert consensus statement on the diagnosis and treatment of paediatric pulmonary hypertension. The European Paediatric Pulmonary Vascular Disease Network, endorsed by ISHLT and DGPK. Heart 2016;102:ii67-ii85.

2 Hansmann G, Apitz C, Abdul-Khaliq H, et al. Executive summary. Expert consensus statement on the diagnosis and treatment of paediatric pulmonary hypertension. The European Paediatric Pulmonary Vascular Disease Network, endorsed by ISHLT and DGPK. Heart 2016;102:ii86-ii100.

3 Lammers AE, Apitz C, Zartner $P$, et al. Diagnostics, monitoring and outpatient care in children with suspected pulmonary hypertension/paediatric pulmonary hypertensive vascular disease. Expert consensus statement on the diagnosis and treatment of paediatric pulmonary hypertension. The European Paediatric Pulmonary Vascular Disease Network, endorsed by ISHLT and DGPK. Heart 2016;102: ii $1-i i 13$

4 Valente AM, Gauvreau K, Egidy Assenza GE, et al. Contemporary predictors of death and sustained ventricular tachycardia in patients with repaired tetralogy of Fallot enrolled in the INDICATOR cohort. Heart 2014;100:247-53. 